\title{
Front Matter: Volume 11286
}

, "Front Matter: Volume 11286," Proc. SPIE 11286, Optical Interconnects XX, 1128601 (10 March 2020); doi: 10.1117/12.2570244

SPIE. Event: SPIE OPTO, 2020, San Francisco, California, United States 


\title{
PROCEEDINGS OF SPIE
}

\section{Optical Interconnects XX}

\author{
Henning Schröder \\ Ray T. Chen \\ Editors
}

4-6 February 2020

San Francisco, California, United States

Sponsored and Published by

SPIE 
The papers in this volume were part of the technical conference cited on the cover and title page. Papers were selected and subject to review by the editors and conference program committee. Some conference presentations may not be available for publication. Additional papers and presentation recordings may be available online in the SPIE Digital Library at SPIEDigitalLibrary.org.

The papers reflect the work and thoughts of the authors and are published herein as submitted. The publisher is not responsible for the validity of the information or for any outcomes resulting from reliance thereon.

Please use the following format to cite material from these proceedings:

Author(s), "Title of Paper," in Optical Interconnects XX, edited by Henning Schröder, Ray T. Chen, Proceedings of SPIE Vol. 11286 (SPIE, Bellingham, WA, 2020) Seven-digit Article CID Number.

ISSN: 0277-786X

ISSN: 1996-756X (electronic)

ISBN: 9781510633353

ISBN: 9781510633360 (electronic)

Published by

SPIE

P.O. Box 10, Bellingham, Washington 98227-0010 USA

Telephone +1 3606763290 (Pacific Time) · Fax +1 3606471445

SPIE.org

Copyright (c) 2020, Society of Photo-Optical Instrumentation Engineers.

Copying of material in this book for internal or personal use, or for the internal or personal use of specific clients, beyond the fair use provisions granted by the U.S. Copyright Law is authorized by SPIE subject to payment of copying fees. The Transactional Reporting Service base fee for this volume is $\$ 21.00$ per article (or portion thereof), which should be paid directly to the Copyright Clearance Center (CCC), 222 Rosewood Drive, Danvers, MA 01923. Payment may also be made electronically through CCC Online at copyright.com. Other copying for republication, resale, advertising or promotion, or any form of systematic or multiple reproduction of any material in this book is prohibited except with permission in writing from the publisher. The CCC fee code is 0277 $786 \mathrm{X} / 20 / \$ 21.00$.

Printed in the United States of America by Curran Associates, Inc., under license from SPIE.

Publication of record for individual papers is online in the SPIE Digital Library.

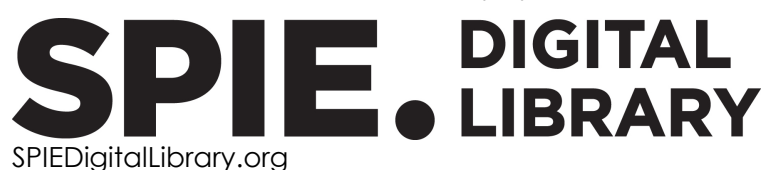

SPIEDigitalLibrary.org

Paper Numbering: Proceedings of SPIE follow an e-First publication model. A unique citation identifier (CID) number is assigned to each article at the time of publication. Utilization of CIDs allows articles to be fully citable as soon as they are published online, and connects the same identifier to all online and print versions of the publication. SPIE uses a seven-digit CID article numbering system structured as follows:

- The first five digits correspond to the SPIE volume number.

- The last two digits indicate publication order within the volume using a Base 36 numbering system employing both numerals and letters. These two-number sets start with 00, 01, 02, 03, 04, 05, 06, 07, 08, 09, OA, OB ... 0Z, followed by 10-1Z, 20-2Z, etc. The CID Number appears on each page of the manuscript. 


\title{
Contents
}

\author{
vii Authors \\ ix Conference Committee
}

\section{OPTICAL INTERCONNECT SYSTEMS}

1128602 Optical interconnects in enterprise and hyperscale datacenters (Invited Paper) [11286-1]

1128603 Market and industrial trends of optical interconnect (Invited Paper) [1 1286-2]

1128604 Intra-satellite optical interconnect space market and technical challenges (Invited Paper) [1 1286-3]

$1128605 \quad$ Nanoseconds photonic integrated switches for optical data center interconnect systems (Invited Paper) [1 1286-4]

1128606 Board-and rack-scale optical interconnection architectures for disaggregated data centers (Invited Paper) [1 1286-47]

HYBRID DEVICE INTEGRATION APPROACHES FOR PIC I

1128607 Photonic plug for scalable silicon photonics packaging (Invited Paper) [1 1286-5]

HYBRID DEVICE INTEGRATION APPROACHES FOR PIC II

11286 OA Detachable 1x8 single mode optical interface for DWDM microring silicon photonic transceivers [11286-8]

\section{FIBER OPTICS AND MICRO-OPTIC ASSEMBLY}

11286 OC Low cutoff G.657-compatible fiber for data center interconnects operating in the 1064 and $1310 \mathrm{~nm}$ windows (Invited Paper) [11286-10]

11286 OD MT-ferrule compatible passive optical coupling for single-mode polymer waveguide in co-packaged optics (Invited Paper) [1 1286-11]

11286 OE Fiber-optic interconnect technologies (Invited Paper) [1 1286-12] 
11286 OF Automated PM-fiber array assembly with high-precision four DOF alignment [1 1286-13]

11286 OG Modular lensed-ferrule solution for single-mode fiber connectors [1 1286-14]

\section{NOVEL OPTICAL WAVEGUIDE AND INTEGRATED INTERCONNECT TECHNOLOGIES}

$11286 \mathrm{OH} \quad$ Bridging the gap: manufacturing optical transceivers in the multibillion-dollar silicon electronics supply chain (Invited Paper) [1 1286-15]

11286 Ol Polymer interposer for efficient light coupling into $3 \mu \mathrm{m}$ silicon-on-insulator waveguides [11286-16]

11286 OK Bending loss improvement and twisting loss studies of flexible multimode polymer waveguides [11286-18]

11286 OL Novel technology for dispensing liquid polymers of a wide viscosity range on a picoliter scale for photonic applications [11286-49]

\section{PICS FOR OPTICAL INTERCONNECTS}

11286 OM Integrated silicon photonics for high-volume data center applications (Invited Paper) [11286-19]

11286 ON Integrated photonic chip to chip interconnection utilising grating coupler technology [1 1286-20]

$1128600 \quad$ High-speed and power-efficient beam-steering using $1 \times 16$ silicon optical phased array with electro-optic phase shifters [11286-21]

11286 OP Building blocks of a scalable and radiation-hardened integrated transmitter unit based on $250 \mathrm{~nm}$ SOI [11286-22]

\section{HYBRID INTEGRATED OPTICAL LINK MODULES I}

$112860 Q \quad$ Design modifications to an existing high-density mid-board optical engine to survive harsh environments (Invited Paper) [1 1286-23]

11286 OR Short reach, low cost silicon photonic micro-transceivers for embedded and co-packaged system integration (Invited Paper) [11286-24]

11286 OS Integrated optical receiver module with demultiplexer for chip-to-chip optical interconnects [1 1286-25]

11286 OT Injection molded low-thermal-expansion multi-fiber ferrule [1 1286-26] 
OPTICAL INTERCONNECT DEVICES I

11286 OU Low-voltage modulators using thin-film lithium niobate (Invited Paper) [1 1286-27]

11286 OW Comparison of three monolithically integrated TIA topologies for $50 \mathrm{~Gb} / \mathrm{s}$ OOK and PAM4 [11286-29]

11286 OX Strain-engineered group IV light sources for photonic-integrated circuits (Invited Paper) [11286-30]

11286 OY A high-performance Echelle grating de-multiplexer based on two stigmatic points and its flat-top solution [1 1286-31]

HYBRID INTEGRATED OPTICAL LINK MODULES II

1128610 Packaging challenges for next-generation high bandwidth opto-electrical switch modules (Invited Paper) [11286-33]

1128612 Ring-assisted Mach-Zehnder interferometer switch with multiple rings per switch element [11286-35]

$1128614 \quad$ Chip level fiber coupling [11286-38]

OPTICAL INTERCONNECT DEVICES II

1128617 Optical interconnects for datacenter links: design and modeling challenges (Invited Paper) [11286-41]

\section{NANOPHOTONIC TECHNOLOGY FOR OPTICAL INTERCONNECTS}

11286 1A High-speed data transmission system using silicon-based optical phased array [11286-44]

11286 1C Metallic optical benches with stamped micro-mirrors for photonic assemblies and optical interconnects (Invited Paper) [1 1286-46] 
Proc. of SPIE Vol. 11286 1128601-6 Downloaded From: https://www.spiedigitallibrary.org/conference-proceedings-of-spie on 26 Apr 2023
Terms of Use: https://www.spiedigitallibrary.org/terms-of-use 


\section{Authors}

Numbers in the index correspond to the last two digits of the seven-digit citation identifier (CID) article numbering system used in Proceedings of SPIE. The first five digits reflect the volume number. Base 36 numbering is employed for the last two digits and indicates the order of articles within the volume. Numbers start with 00, 01, 02, 03, 04, 05, 06, 07, 08, 09, 0A, 0B...0Z, followed by 10-12, 20-2Z, etc.

Aalto, Timo, 01

Ahmed, Abu Naim R., OU

Alexoudi, T., 06

Alippi, A., OF

Alreesh, Saleem, 17

Amano, T., OD

Andrade, Hector, OW

Bamiedakis, N., OK

Bellman, Robert A., OT

Berger, M., OF

Bickham, Scott R., OC

Blanchette, Guillaume, 04

Blum, Robert, OM

Boyer, Nicolas, 10

Brecher, C., OF

Buckwalter, James F., OW

Burt, Kevin, $O Q$

Calabretta, N., 05

Chen, Yang, $1 \mathrm{C}$

Childers, Darrell, OA, OT

Chu, D., OK

Coggi, Victor, OG

Cyr, Elaine, 10

Dockchoorung, Woraphat, OT

Dris, Stefanos, 17

Eisenblätter, L., OP, OY

Fiorentino, Marco, OA

Fortier, Paul, 10

Fotiadis, K., 06

Guo, Xiaotao, 05

Gustavsson, Johan S., OC

Haag, S., OF

Han, Kyeongjin, 1A

Harjanne, Mikko T., Ol

Hastings, DJ, OT

Heimala, Päivi, 01

Hii, King-Fu, $1 \mathrm{C}$

Hiltunen, Marianne, $\mathrm{Ol}$

Hirokawa, Takako, OW, 12

Hoeren, M., OF

Hulme, Jared, OA

Israel, Abraham, 07

Janta-Polczynski, Alexander, 10

Kang, Geumbong, 00

Käpplinger, I., 14

Karnick, D., OP, OY

Klein, J., OL

Koltchanov, Igor, 17

Kühner, T., OP, OY
Kurata, Kazuhiko, OR

Kurtz, Dan, OA

LaCroix, Louis, $0 Q$

Langlois, Richard, 10

Larsson, Anders, OC

Lee, Bernard, 03

Lee, Dae-Seong, 00

Lee, Raymond, $0 \mathrm{Q}$

Lengyel, Tamás, OC

Li, Chao, 07

Li, Ming-Jun, $O C$

Lo, Patrick Guo Qiang, 07

Maharry, Aaron, OW

Malhouitre, Stephane, 07

Marcoccia, Roberto R., $\mathrm{OH}$

Marin, Esteban, OT

Marx, S., OL

Mathai, Sagi, OA

Menezo, Sylvie, 07

Mercante, Andrew J., OU

Mishra, Snigdharaj K., OC

Mitsolidou, C., 06

Möller, Ch., 14

Moralis-Pegios, M., 06

Mourgias-Alexandris, G., 06

Müller, Tobias, OF

Nagase, Ryo, OE

Nam, Donguk, OX

Neckermann, K., 14

Nelan, Sean, OU

Neukirch, Ulrich W., OT

Nguyen, Nga T. H., OS

Noriki, A., OD

O'Brien, Peter, ON

Ortlepp, H.-G., 14

Ortlepp, T., 14

Pan, Bitao, 05

Panotopoulos, George, OA

Park, Hyo-Hoon, 00, 0S, IA

Pascar, Leonid, 07

Pashkova, Tatiana, ON

Penty, R. V., OK

Piehler, David, 02

Pitris, S., 06

Pitwon, Richard, OR

Pleros, N., 06

Prather, Dennis W., OU

Prifti, Kristif, 05

Reid, Benoit, 04 
Rhee, Hyun-Woo, $1 \mathrm{~A}$

Rhim, Jinsoo, OA

Richter, André, 17

Rolston, David, 04

Rosenberg, Paul, OA

Saeidi, Mitra, 12

Saleh, Adel A. M., 12

Sanadgol Nezami, Mohammadreza, 04

Sangirov, Jamshid, OS

Schmidt, Theodore J., $\mathrm{OH}$

Schneider, M., OP, OY

Schow, Clint L., OW, 12

Schröder, H., OL

Seyedi, Ashkan, OA

Shi, F., OK

Shi, Shouyuan, OU

Simon, Stefan, OW

Simpanen, Ewa, OC

Smith, Stephen Q., OT

Sokolov, Eugene, 17

Sun, Peng, OA

Taha, Hesham, 07

Tan, Michael, OA

Tandon, Pushkar, OC

Terzenidis, N., 06

Theogarajan, Luke, 12

Tonini, Andrea, OG

Ukaegbu, Ikechi Augustine, OS

Ulfan, Faivush, 07

Valenzuela, Luis, OW

Vallance, R. Ryan, 1C

Van Vaerenbergh, Thomas, OA

Vehmas, Tapani, OI

Vyrsokinos, K., 06

Wachholz, P., OL

Wälchli, Ben, 0 I

Wang, Ying, 07

Weber, D., OL

Weber, M., OP, OY

White, I. H., OK

Wilmart, Quentin, 07

Wolf, J., OL

Xue, Xuwei, 05

Yan, Fulong, 05

Yao, Peng, OU

Yevseyenko, Dmitry, 17

Yoon, Hyeonho, $0 \mathrm{O}$

You, Jong-Bum, IA

Zhang, Y., OP, OY

Zontar, D., OF 


\section{Conference Committee}

Symposium Chairs

Sailing He, KTH Royal Institute of Technology (Sweden) and Zhejiang University (China)

Yasuhiro Koike, Keio University (Japan)

Symposium Co-chairs

Connie J. Chang-Hasnain, University of California, Berkeley (United States)

Graham T. Reed, Optoelectronics Research Centre, University of Southampton (United Kingdom)

Program Track Chairs

Yakov Sidorin, Quarles \& Brady LLP (United States)

Jean-Emmanuel Broquin, IMEP-LAHC (France)

Conference Chairs

Henning Schröder, Fraunhofer-Institut für Zuverlässigkeit und Mikrointegration IZM (Germany)

Ray T. Chen, The University of Texas at Austin (United States)

Conference Program Committee

Maggie Yihong Chen, Texas State University (United States)

Darrell Childers, US Conec Ltd. (United States)

Hamed Dalir, Omega Optics, Inc. (United States)

Alan F. Evans, Corning Incorporated (United States)

Ruth Houbertz, Multiphoton Optics GmbH (Germany)

Marika P. Immonen, TTM Technologies, Inc. (Finland)

Takaaki Ishigure, Keio University (Japan)

Mikko Karppinen, VTT Technical Research Centre of Finland Ltd. (Finland)

Christian Koos, Karlsruher Institut für Technologie (Germany)

Tobias Lamprecht, vario-optics ag (Switzerland)

Matthias Lorenz, AEMtec GmbH (Germany)

Christopher T. Middlebrook, Michigan Technological University

(United States)

Bert-Jan Offrein, IBM Research - Zürich (Switzerland)

Hyo-Hoon Park, KAIST (Korea, Republic of)

Ignazio E. M. Piacentini, ficonTEC Service GmbH (Germany)

Nikos Pleros, Aristotle University of Thessaloniki (Greece) 
Richard C. A. Pitwon, University of St. Andrews (United Kingdom)

Jie X. Qiao, Rochester Institute of Technology (United States)

Brandon W. Swatowski, Dow Corning Corporation (United States)

Dave J. Thomson, Optoelectronics Research Centre (United Kingdom)

Huiping Tian, Beijing University of Posts and Telecommunications (China)

Alan X. Wang, Oregon State University (United States)

Ian H. White, University of Cambridge (United Kingdom)

Chris Q. Wu, Corning Incorporated (United States)

\section{Session Chairs}

1 Optical Interconnect Systems

Henning Schröder, Fraunhofer-Institut für Zuverlässigkeit und Mikrointegration IZM (Germany)

2 Hybrid Device Integration Approaches for PIC I

Ray T. Chen, The University of Texas at Austin (United States)

3 Hybrid Device Integration Approaches for PIC II

Ray T. Chen, The University of Texas at Austin (United States)

4 Fiber Optics and Micro-Optic Assembly

Alethea Vanessa Zamora Gomez, Fraunhofer-Institut für Zuverlässigkeit und Mikrointegration IZM (Germany)

5 Novel Optical Waveguide and Integrated Interconnect Technologies Ruth Houbertz, Multiphoton Optics GmbH (Germany)

$6 \quad$ PICs for Optical Interconnects

Maggie Yihong Chen, Texas State University (United States)

$7 \quad$ Hybrid Integrated Optical Link Modules I

Jie X. Qiao, Rochester Institute of Technology (United States)

8 Optical Interconnect Devices I

Torsten Vahrenkamp, ficonTEC Service GmbH (Germany)

9 Hybrid Integrated Optical Link Modules II

Jie Qiao, Rochester Institute of Technology (United States)

10 Optical Interconnect Devices II

Hamed Dalir, Omega Optics, Inc. (United States)

11 Nanophotonic Technology for Optical Interconnects

Darrell Childers, US Conec Ltd. (United States) 